\title{
Economic feasibility of freeze-drying to obtain powdered fruit
}

\section{Camacho, M.M.a ; Casanova, M.A.a ; Fenollosa, L.b; Ribal, J. ${ }^{\mathbf{b}}$; Martínez-Lahuerta,} J.J. ${ }^{c}$; Martínez-Navarrete, $\mathbf{N}^{{ }^{a^{*}}}$

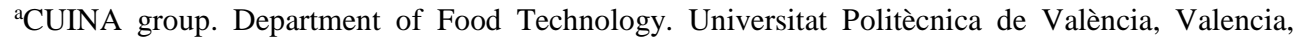
Spain

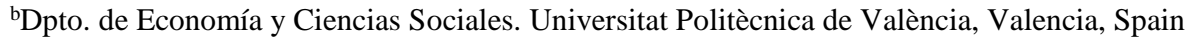
${ }^{\mathrm{c}}$ Centre Auxiliar Juan Llorens, Valencia, Spain

* E-mail of the corresponding author: nmartin@tal.upv.es

\begin{abstract}
Fruit is a highly valuable food whose consumption should be encouraged. In addition, it would be desirable to design processes and products to channel the surplus and take advantage of the post-harvest losses that limit its fresh marketing. Freeze-drying is a known industrial process that permits the obtaining of high quality products, despite having always been labeled as very expensive. In this study, the economic feasibility of freeze-drying to obtain powdered fruit has been proven, as it yields a product more than twice as cheap as when obtained by spray-drying, recognized for its low cost.
\end{abstract}

Keywords: freeze-drying; spray-drying; economic profitability; production costs 


\section{Introduction}

The influence of climatic conditions on fruit production makes seasonal surpluses an inherent characteristic of the sector. In addition, they are highly perishable foods that in the phases of post-harvest, storage and transport lose a part of their properties, which limits their fresh consumption. Last but not least, a great deal of fruit is lost both in wholesale and household waste. In this sense, preventing the waste of fruit becomes a challenge for society. For this reason, it seems appropriate to propose viable new processed fruit products, so that their surpluses are used and the consumption of this food is promoted. Freeze-drying is a dehydration technique that allows high quality products to be obtained, ones which are highly stable and very easy to handle, in terms of their greater ease of transport and storage. However, this process has always been labeled as a very expensive one. Spray-drying also allows high quality powder to be obtained from food. An important difference between the two processes is that freeze-drying may take advantage of all the edible part of the fruit in the form of a puree, whereas the liquidized fruit is required for the spray-drying, with the consequent generation of a greater quantity of by-products. In both cases, the obtained powdered product will be of great microbiological, chemical and biochemical stability. However, it could present important physical problems related to the glass transition of its amorphous matrix. In the rubbery state, the structural collapse of the amorphous matrix occurs. In the case of powdered products this leads to phenomena such as stickiness development ${ }^{[1]}$. To avoid this problem, the incorporation of high molecular weight biopolymers as stabilizers is suggested ${ }^{[2]}$. In this context, the research team involved in this study has worked towards obtaining powdered fruit by freeze-drying and spraydrying. In this sense, the amount of biopolymers to be added has been optimized in order to obtain a powdered grapefruit with a high product yield and also of high physical, nutritional and functional quality ${ }^{[3]}$. The obtained results allow freeze-drying to be put forward the propose as a better technique than spray-drying for the purposes of obtaining high quality fruit products. However, from the perspective of the commercial viability of new products, it is essential to consider the final consumer cost, dependent on both the initial investment and the cost associated with its production. Thus, it seems necessary to perform an economic analysis by comparing both processes, which was the objective of the present study.

\section{Materials and Methods}

The industrial processes considered in this study were freeze-drying and spray-drying. The raw material needed to carry out both of them were grapefruit (Citrus paradisi, var. Star Ruby), gum Arabic (GA, Scharlab) and bamboo fiber (BF, Vitacel@ BAF 200). Water was also needed in the case of spray-drying. 


\subsection{Quantification of the initial investment}

To quantify the initial investment, the machinery necessary to implement both processes was considered. To select the different equipment needed in each case, the estimated product production volume was considered, as were its technical characteristics and price. In order to propose the necessary amount of powder product, it was assumed that its final use was to be rehydrated for the purposes of obtaining a fruit juice. In order to fix the fruit juice to be obtained, the average annual production of refrigerated juice by a company located in the Valencian Community between 2009 and 2014, 87 t, was selected as a reference value. The amount of raw material to be processed by each process was calculated taking into account the amount of powdered product needed to obtain the $87 \mathrm{t}$ of rehydrated juices ${ }^{[4]}$. The most appropriate and economical machinery was selected from what was proposed by the different manufacturing companies and consequently dimensioned.

\subsection{Production cost analysis}

Variable and fixed production costs were calculated. Variable costs depend on the quantity of product produced and include raw material, personnel costs, electrical costs and the management of by-products (grapefruit peel, pulp and water removed from fruit). The fixed costs are independent of the quantity produced and include maintenance and amortization, calculated according to the straight-line method, which consists of dividing the machinery acquisition value by the useful life according to the technical information.

\subsection{Investment analysis}

To calculate the minimum cost of the product, a static analysis was carried out, comparing income and expenses and equalling the profit to zero. On the other hand, to calculate the price that provides a minimum profitability, a dynamic analysis was carried out. A discount rate calculated as the sum of a risk-free rate plus a risk premium was used. As a risk-free rate, the average of the 10-year bond yield on the primary market, issued by the Spanish government in 2016, was taken, this being $1.6 \%$. As a risk premium, 5.5\% was considered. These data were obtained from a study carried out in 2015 on 41 countries ${ }^{[5]}$. Therefore, the assumed discount rate was $7.1 \%$. 


\section{Results and discussion}

\subsection{Selected machinery}

To prepare the sample for freeze-drying, an industrial automatic-feeding knife peeler (1000 - 1100 grapefruit/h). was selected. The selected crusher was a hammer mill with vertical axis $(1500 \mathrm{~kg} / \mathrm{h})$. The grinding process does not generate waste because the hammers of the mill disintegrate the particles until they reach the right size. For the sample to be spraydried, a high-performance centrifugal liquidizer was chosen. This machine guarantees a quick process so that product oxidation is minimized. In this case, obtaining the liquidized product generates a by-product in the form of grapefruit pulp. According to the technical characteristics of the selected equipment, for each kilo of fruit that enters the liquidizer, $0.383 \mathrm{k}$ of pulp and $0.617 \mathrm{k}$ of liquidized fruit are obtained. The obtained by-product has been supposed to be commercialized. For both processes, a horizontal shaft vane mixer was selected to incorporate the biopolymers and the water into the puree or the liquidized fruit, thus achieving a better product homogenization.

Due to the characteristics of the sample and the estimated production volume, two freezedryers based on conduction heat transfer and a centrifugal spray-dryer were chosen. In the case of freeze-drying, this process was estimated to require 24 hours of operation to sublimate the water correctly ${ }^{[3]}$. For this reason, two freeze-dryers have been considered working in parallel so that they can operate continuously every day. In the case of the spray-dryer, as the one that most suited the required volume of powder production actually has a higher production capacity, the corresponding operational costs were refitted to the latter in order not to discriminate by oversizing it.

For the packaging of the obtained powdered products, an opaque material of very low water vapour permeability was selected, so as to prevent the humidification and destruction of photosensitive compounds, such as vitamin $\mathrm{C}$. The selected packaging machine allows different formats to be used. A mono-dose (44.5 $\mathrm{g}$ of powder) and another one for the HORECA channel (379 $\mathrm{g}$ of powder) were chosen. These quantities respond to the powder needed to obtain a glass of juice of $125 \mathrm{~cm}^{3}$ and $1100 \mathrm{~cm}^{3}$, respectively.

\subsection{Production costs}

To obtain the $87 \mathrm{t}$ of juice assumed as the reference value, 26,551 $\mathrm{kg}$ of powder obtained either by freeze-drying or by spray-drying need to be rehydrated. The corresponding raw material required (grapefruit, GA, FB and water) and its cost was calculated by taking into account the yield of each selected machine and our own preliminary experiences ${ }^{[4]}$ (Table 
1). Assuming the price of the raw materials, according to commercial data $(0.73 € / \mathrm{kg}$ grapefruit, $78.9 € / \mathrm{kg} \mathrm{GA}, 2.5 € / \mathrm{kg} \mathrm{FB}$ and $0.0025 € / \mathrm{kg}$ water), the total annual costs were $771,309.27$ and 2,704,407.92 $€$ for freeze-drying and spray-drying, respectively. As can be observed in Table 1, the total cost of the raw material needed for the spray-drying process is 3.5 times higher than for freeze-drying. The former has a much lower powder yield than the latter, especially due to the powder loss that occurs in the spray-dryer itself. A significant part of the powder remains adhered to the walls and nozzle of the equipment. For this reason, the fruit cost of spray-drying is 6 times that of freeze-drying.

Table 1. Daily needs (Dn) and annual cost (Ac) of raw materials for freeze-drying (FD) and spraydrying $(S D)$ processes

\begin{tabular}{lllll}
\hline & Grapefruit & GA & BF & Water \\
\hline Dn FD (kg/day) & 654,1 & 20,60 & 2,94 & 147 \\
Dn SD (kg/day) & 3885 & 57,03 & 28,52 & 1425,8 \\
Ac FD (€) & $174.281,76$ & $593.207,37$ & $2.685,85$ & 134,29 \\
Ac SD $(€)$ & $1.035 .088,26$ & $1.641 .998,13$ & $26.020,51$ & $1.301,03$ \\
\hline
\end{tabular}

The total cost in machinery for each technology is presented in Table 2. The initial investment in machinery is 1.4 times higher for the spray-drying than for the freeze-drying process, even though a freezer is used for freeze-drying and two freeze-dryers are required working in parallel. The spray-dryer and the 2 freeze-dryers account for $96 \%$ and $94 \%$ of the total machinery investment in each process.

Table 2. Variable and fixed production costs and product price in monodose and HORECA format

\begin{tabular}{lll}
\hline & Freeze-drying & Spray-drying \\
\hline Electric costs $(€)$ & $354.861,65$ & $41.955,41$ \\
Raw material costs $(€)$ & $770.309,27$ & $2.704 .407,92$ \\
By-products costs $(€)$ & $-658,98$ & $-17.777,56$ \\
Personnel expenses $(€)$ & $150.970,26$ & $368.494,39$ \\
Amortization $(€)$ & $55.266,67$ & $73.355,56$ \\
Maintenance $(€)$ & $97.500,00$ & $130.800,00$ \\
Investment in machinery $(€)$ & 955.000 & 1.288 .000 \\
Total cost $(€)$ & $1.428 .248,86$ & $3.301 .235,72$ \\
Static price mono dose $(€ / \mathrm{ud})$ & 2,39 & 5,53 \\
Static price HORECA $(€ / \mathrm{ud})$ & 17,94 & 41,47 \\
\hline
\end{tabular}

The electrical cost is usually one of the highest in this type of industry. To calculate the total electrical cost, it is necessary to calculate the energy cost due to electricity 
consumption and the contracted power cost that is necessary for the machines to operate. To calculate the electrical cost, the consumption of each selected machine, the number of machines needed in each stage, the working hours per machine and the power were considered (Table 2). For these calculations, an electricity tariff was selected which considers different prices of the kWh depending on whether it is a weekday or weekend and also on the operating hours of the day. When comparing the electrical costs of both processes, it can be affirmed that freeze-drying has an annual consumption that is 8.5 times higher than spray-drying.

Amortization is an economic term referring to the distribution over time of a durable value, with a maximum linear coefficient, or a maximum percentage regulated in the accounting that is allowed to amortize annually, of $12 \%$. The annual amortization has been calculated based on the quotient between the price of the machinery and its useful life. The useful life was considered to be what was specified in the technical characteristics of each machine, except in the case of the freeze-dryer and the spray-dryer. As there were no data available for these equipment, the accounting useful life was considered, this being 18 years according to the provisions of Law 27/2014, of November 27. The amortization of the spray-dryer was 1.3 times higher than that of the freeze-dryer (Table 2). This is due to the fact that the machinery used in the spray-drying technique has a higher cost than what was used for freeze-drying. Again, the freeze-dryer and the spray-dryer are the machines with the highest cost in amortization, due to the much higher acquisition cost of these equipment.

As regards by-products, there are two that could be exploited by other companies: the grapefruit peel after both processes and the pulp that is separated when liquidizing in the case of spray-drying. In these cases, it has been considered that the peel and pulp can be sold at 0.015 and $0.025 € / \mathrm{kg}$, respectively, according to the average price provided by various companies in the sector. Taking into account the volume of the generated byproducts, their sale would provide the income that is observed in Table 2. Thus, the only thing that would cause a real cost would be the discharge of water. Therefore, there is really no a net cost in the management of by-products, but an income that, in the case of spraydrying, is 26 times higher than the income obtained from the management of by-products generated with the freeze-drying.

\subsection{Minimum price of the product: static analysis}

The total annual cost of the spray-drying technique is 2.3 times higher than that of freezedrying (Table 2). Figure 1 shows the percentage represented by each item over the total cost. The maintenance and amortization expenses in the case of spray-drying are 1.34 and 1.32 times, respectively, those of freeze-drying. This is because of the greater initial 
investment in machinery. In adition, the personnel cost needed to obtain the spray-dried powder is 2.4 times that of the freeze-dried, while the electrical cost for the spray-drying process is 8.5 times lower than for freeze-drying. Such a great difference is due to the need for a vacuum in the case of freeze-drying and the operating time per $\mathrm{kg}$ of the fresh product. The highest cost in both processes is that of the raw material, which is $54 \%$ and $82 \%$ for freeze-drying and spray-drying, respectively. This difference is due to the low yield of the latter when working with fruit.

The static price, or the final product cost, in the case of the spray-dried powder is 2.31 times higher than that of the freeze-dried (Table 2). It was calculated for the two formats with which the powder product was considered to be packaged.

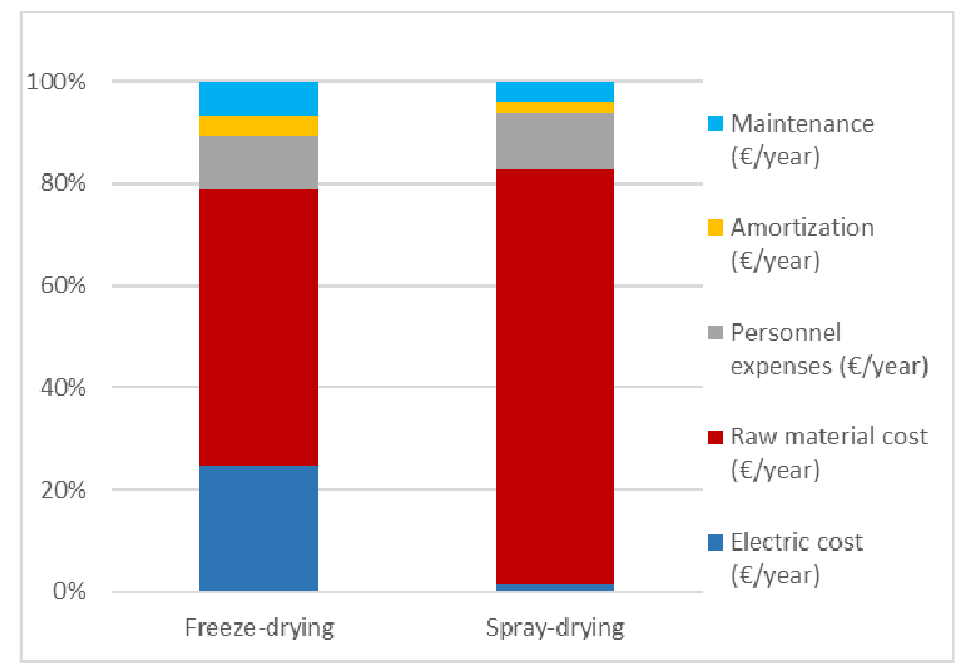

Fig. 1 Percentage of the cost represented by each of the items considered, calculated as related to the total cost of the freeze-drying and spray-drying processes.

\subsection{Price of the product that provides a minimum profitability: dynamic analysis}

To calculate this price, the internal rate of return (IRR) and the net present value (NPV) were calculated for both the freeze-drying and spray-drying processes. To this end, it is necessary to know the different cash flows that are calculated, in turn, from the extraordinary payments (renovations in machinery originally planned and that are taken as $5 \%$ of the value of the machine when new) and ordinary charges (capital inflows from the sale of the product). In this case, we have considered the NPV null hypothesis in order to calculate the price that will result in IRR being 7, which is the same as looking for the price that will obtain a $7 \%$ profitability. Thus, for freeze-dried powder, the price is 2.84 and 
21.25€ per unit in the mono-dose and HORECA formats, respectively. The corresponding prices for the spray-dried powder is $6.13 €$ and $45.95 €$ per unit.

\section{Conclusions}

This economic study shows that the application of freeze-drying to obtain powdered fruit provides a product at a cost that is less than half that of spray-drying, a process known for its low cost. The lower cost of freeze-drying is mainly due to the poor yield of spray-drying in the case of fruit processing. Thus, although the electrical costs are 8.5 times higher in the case of freeze-drying, both the cost in raw material and the initial investment in machinery, personnel costs, maintenance and amortization are higher in the case of spray-drying. So much so that the estimated price for a dose of grapefruit powder equivalent to a piece of fruit, for example, would be 2.84 versus 6.13 euros depending on whether it was obtained by freeze-drying or by spray-drying, respectively.

\section{Acknowledgements}

The authors thank the Ministerio de Economía y Competitividad for the financial support given through the Project AGL 2012-39103.

\section{References}

[1] Roos, Y. Characterization of Food Polymers Using State Diagrams. Journal of Food Engineering 1995, 24(3), 339-360.

[2] Telis, N.V.; Martínez-Navarrete, N. Biopolymers used as drying aids in spray drying and freeze drying of fruit juices and pulps. In: Biopolymer Engineering in Food Processing. V. Telis (Ed.). CRC Press. Taylor \& Francis Group, Boca Raton, FL. 2012, 279-326.

[3] Agudelo, C.; Igual, M.; Camacho, M.M.; Martínez-Navarrete, N. Effect of process technology on the nutritional, functional and physical quality of grapefruit powder. Food Science and Technology International, 2017, 23(1), 61-74.

[4] Casanova, M.A. Estudio de viabilidad para la comercialización de fruta en polvo. Trabajo Final de Grado E.T.S.I.A.M.N; Universitat Politècnica de Valencia. Spain, 2014.

[5] Fernandez, P.; Ortiz, A.; Acin, I. F.. Discount Rate (Risk-Free Rate and Market Risk Premium) used for 41 countries in 2015: a survey. IESE Business School, 2015, 1, 3-20. 